\title{
Comparison between traditional and goal directed perfusion in cardiopulmonary by-pass. A differential cost analysis in US
}

\author{
Massimiliano Povero ${ }^{1}$, Lorenzo Pradelli ${ }^{1}$ \\ AdRes Health Economic and Outcome Research, Torino, Italy
}

\begin{abstract}
OBJECTIVES: High oxygen delivery (DO2) during cardiopulmonary bypass (CPB) is associated with better renal outcome in cardiac surgery. Traditional perfusion (TP) techniques, targeted on body surface area and CPB temperature, achieves high DO2 in about $50 \%$ of the cases while a goal directed perfusion (GDP) approach can lead to more than $90 \%$ of cases achieving high DO2 with a consequent reduction in Acute Kidney Injury (AKI) rate of about $40 \%$. Aim of this study is to perform an economic evaluation of GDP strategy with respect to TP in US. METHODS: A Discrete Event Simulation model was developed to compare TP and GDP strategy in patients undergoing CPB. The patient's pathways from operation to discharging from hospital was simulated: AKI incidence, in-hospital mortality, hospital length of stay, transfusions were correlated to probability to achieve high DO2 target using published correlations. National perspective was adopted to calculate costs associated to each event while GDP strategy was exploited the introduction of Sorin Heartlink (HL) Card/GDP Card and Sorin Connect (electronic data management system). RESULTS: GDP strategy saved more than 3 days in hospital and $11 \%$ of AKI episodes. The cost-saving is $\$ 3,137$ (95\% CI: 1,122-4,951); the cost of HL Card/GDP Card+Connect (\$ $180,95 \%$ CI: $113-249)$ is more than offset by savings in hospital stay that result the main driver in cost ( $\$ 3,222,95 \%$ CI: 1,235-4,950). Deterministic sensitivity analysis shows that the total savings are mainly influenced by nadir haematocrit during CPB and hospital LOS/cost per day both in ICU and in ward. CONCLUSIONS: GDP seems to improve significantly the main outcomes related to CPB surgery, when compared to TP techniques. Additional costs due to perform GDP strategy have no impact on the total cost since completely offset by the savings in hospital cost.
\end{abstract}

\section{Keywords}

Cardiopulmonary bypass; Traditional perfusion; Goal directed perfusion

\section{INTRODUCTION}

Oxygen delivery (DO2) and carbon dioxide production $(\mathrm{VCO} 2)$ during cardiopulmonary bypass (CPB) are associated with renal outcome in cardiac surgery $[1,2]$. Nadir DO2 below the threshold of $262-272 \mathrm{ml} / \mathrm{min} / \mathrm{m}^{2}$ and $\mathrm{DO} 2 / \mathrm{VCO} 2$ ratio lower than 5.0 are linked to an increased incidence of acute kidney injury (AKI) after cardiac operations [1].

Data collected in [2] shows that 693 patients reached the DO2 target among 1,048 patients undergoing $\mathrm{CPB}$ with a traditional perfusion strategy. Such patients were divided in two groups according to HCT level; as anticipated by physiology (see Appendix A), results show that the probability to achieve the $\mathrm{DO} 2$ goal resulted higher among patients with HCT $>26 \%$ (640 of 753 patients) than in patients with $\mathrm{HCT}<26 \%$ (53 of 293 patients).
Sorin HeartLink ${ }^{\mathrm{TM}}$ is the first and unique perfusion system automatically integrating perfusion data, patient parameters and product information to enable implementation of Goal Directed Perfusion (GDP) adapting adequacy of perfusion to the patient.

Hypothesis of the Goal dIrected perFusion Trial (GIFT) protocol is that a GDP approach can raise this percentage to more than $90 \%$ of cases, with a consequent reduction in the AKI rate of about $40 \%$ [3].

Aim of this project is to perform an economic evaluation of the consequences of GDP strategy in US, through the construction, validation, and analysis of an ad hoc simulation model based on estimation of goal-achievement rate (DO2 value over the threshold of $272 \mathrm{ml} / \mathrm{min} / \mathrm{m}^{2}$ ) with and without GDP and the correlations between main clinical out-
Corresponding author Massimiliano Povero m.povero@adreshe.com

\section{Disclosure}

This study was funded by Sorin Group. 
comes (AKI incidence, in-hospital mortality, hospital length of stay, transfusions) and DO2 over the critical threshold. All possible patient pathways during the peri- and post-operative period were considered in order to estimate management of adverse events, hospital stay and related resource consumption costs.

\section{METHODS}

\section{Model structure}

A patient-level simulation model was developed incorporating baseline outcomes rates and comparative efficacy data from published literature, and US hospital cost data.

The model simulates two treatment alternatives for patients undergoing CPB: traditional perfusion (TP - targeted on BSA and CPB temperature) and goal-directed perfusion (GDP - specifically aimed at maintaining DO2 over the critical threshold). GDP is allowed by an integrated monitoring system (Sorin $\mathrm{GDP}^{\mathrm{TM}}$ Monitor) displaying DO2 and $\mathrm{DO} 2 / \mathrm{VCO} 2$ values in a quasi-continuous mode: the perfusionist can immediately react to lowering DO2 by increasing pump flow, as a primary intervention.

The model was structured as a decision analytic Discrete Event Simulation (DES); in a DES changes in the individuals' state are modelled over time, in terms of events that occur and their consequences. This strategy is preferable to the Markov methodology in representing clinical conditions that are neither chronic nor characterized by recurrent events occurring at fixed time intervals, like in patients analysed here.

According to this technique, each iteration of the simulation represents a unique patient,

\begin{tabular}{lc}
\hline \multicolumn{1}{c}{ Event } & Depending on \\
\hline During CPB surgery & \\
Reaching DO2 target & HCT, $\mathrm{P}^{*}$ \\
PRCs transfused & $\mathrm{HCT}$ \\
Renal disease (AKI) & $\mathrm{DO} 2$ \\
AKI needing of RT & $\mathrm{DO} 2$ \\
In ICU (post-CPB) & \\
LOS & $\mathrm{DO} 2$ \\
PRCs transfused & $\mathrm{HCT}$ \\
Death & $\mathrm{AKI}$ \\
In ward & \\
LOS & $\mathrm{DO} 2$ \\
\hline
\end{tabular}

Table I. Events evaluated during the simulation

* perfusion strategy (traditional or GDP)

$\mathrm{AKI}=$ acute kidney injury; $\mathrm{HCT}=$ nadir haematocrit; $\mathrm{LOS}=$ length of stay; PRCs = packed red cells who is sent simultaneously through both treatment arms of the model. The parameters of each iteration are drawn from their distributions, allowing representation of the individual variability in the outcomes of the simulated population. The simulation steps are qualitatively common for both treatment arms, which however differ in the probability of the events considered, and for the time-toevent distributions.

The patient's pathway (Table I) is detailed below.

During CPB:

- Each patient is characterized by sampling a nadir haematocrit (HCT) value; this is independent of the perfusion strategy considered.

- According to the HCT level ( $>$ or $<26 \%$ ), patients reach DO2 target with a probability that depends on the perfusion strategy.

- According to transfusion protocol (depending on HCT level) patients can receive packed red cells (PRCs).

- Patients are at risk of renal adverse events:

- AKI, with probabilities depending on DO2 level (target reached or not).

- Renal failure needing RT (only for patients experiencing AKI) with probabilities that also depend on DO2 level (target reached or not).

Post CPB:

- During the post-operative period, patients can die with probability that depends on renal complications (AKI or not) and can receive PRCs.

- Post-operative length of stay (LOS) in ICU depends on DO2 level during CPB.

- LOS in ward (only for patients discharged alive from ICU) depends on DO2 level during CPB.

The hospital perspective was adopted when calculating costs associated to each state and to evaluate the differential economic impact of GDP vs TP. Only potentially differential costs were considered in the analyses so the total cost calculated in the simulation represents the sum of these cost items.

Half-cycle correction was applied to LOS in ICU and to number of PRCs post-CPB for patients dying in ICU.

\section{Input parameters}

\section{Clinical parameters not depending on DO2 level}

The lowest haematocrit during CPB was taken from a retrospective analysis on data from 5,000 consecutive cardiac operations with CPB performed on adults (1994 to 2000) [4]. For each modelled patient, a nadir 


\begin{tabular}{|c|c|c|c|}
\hline Parameters & \multicolumn{2}{|c|}{ Values } & Sources \\
\hline Nadir HCT on CPB (\%) [mean (SD)] & \multicolumn{2}{|c|}{$21.4(4.2)$} & {$[4]$} \\
\hline Probability to achieve DO2 goal (\%) & High HCT & Low HCT & \\
\hline With TP & 85 & 18 & {$[2]$} \\
\hline \multirow[t]{2}{*}{ With GDP } & 100 & 90 & Elaborated from [3] \\
\hline & With AKI & w/o AKI & \\
\hline Operative mortality (\%) & 11.9 & 1.2 & Elaborated from [5], see Table IA in Appendix B \\
\hline Transfusion protocol & During CPB & Post CPB (in ICU) & \\
\hline PRC units [mean (SD)] & $1.7(2.0)$ & $1.1(1.4)$ & Elaborated from $[1]^{\star},[6]$ \\
\hline Transfusion rate (\%) & & & [3] \\
\hline - $\mathrm{HCT}<19 \%$ & 100 & 100 & Mandatory \\
\hline - $\mathrm{HCT}<24 \%$ & 50 & 50 & PRC allowed \\
\hline - $\mathrm{HCT}<30 \%$ & 0 & 25 & $\begin{array}{l}\text { Admitted based on physician's judgment only } \\
\text { during CPB }\end{array}$ \\
\hline - Other & 0 & 0 & PRC prohibited \\
\hline
\end{tabular}

Table II. Clinical input values not depending on DO2 level, directly

*Gamma distribution was fitted on median (1) and interquartile range (2) of PRCs to estimate mean and SD used in the model

HCT value was sampled from a gamma distribution fitted on mean and SD reported in the article (Table II).

Probability to achieve the DO2 target with traditional perfusion was elaborated from [2] as explained in the Introduction while for the GDP strategy we supposed the $100 \%$ of success for patients with nadir HCT $>26 \%$ during $\mathrm{CPB}$ and $90 \%$ otherwise (Table II).

The relationship between AKI incidence and post-operative mortality was investigated, using logistic regression modelling, in a multicentre cohort study on 3,500 adult patients undergoing cardiac surgery at 7 hospitals during 2004 [5]. Three thresholds of AKI were selected to evaluate mortality rates: $>25 \%,>$ $50 \%$, and $>75 \%$ decrease in estimated glomerular filtration rate (eGFR) within 1 week of surgery. Since in this study eGFR was estimated using the Cockcroft-Gault formula, an eGFR decrease greater than 33\% coincides with the definition of AKI in the GIFT protocol (i.e. $>50 \%$ increase in serum creatinine, see Appendix B for calculation details). Assuming that patients with eGFR decrease $25-50 \%$ were uniformly distributed (i.e. one third between $25-33 \%$ and 2 thirds between $33-50 \%$ ), post-operative mortality results in $11.9 \%$ and $1.2 \%$ for patients experiencing and not experiencing AKI, respectively (Tabel II).

During $\mathrm{CPB}$, according to current practice and the GIFT protocol, transfusions are mandatory below a HCT of $18 \%$ and generally prohibited for an HCT $>21 \%$. However, based on the individual judgment that the pa- tient is actually in need for packed red cells, transfusions are allowed between an HCT of $22 \%$ and $24 \%$. Transfusions are always prohibited for an HCT $>24 \%$. After CPB, transfusions are mandatory if $\mathrm{HCT}<18 \%$, allowed for HCT between $19 \%$ and $23 \%$ and generally prohibited otherwise. However for HCT between $24 \%$ and $30 \%$, transfusions are admitted based on physician's judgment. In order to simulate this uncertainty in the model, we chose a probability of $50 \%$ to be transfused for allowed transfusions and $25 \%$ for transfusions admitted based on physician's judgment.

Numbers of PRCs transfused during CPB and after CPB (in ICU) were sampled from gamma distributions fitted on mean and SD reported in [1] and [6], respectively (Table II).

\section{Clinical input parameters depending on DO2 level}

A retrospective analysis of data prospectively collected at two different institutions [1] shows that hospital and ICU length of stay (LOS) and renal disease (AKI) are correlated with the DO2 level during CPB (Table III). Length of stay in ward was calculated starting from hospital and ICU LOS according to mortality and AKI rate; in fact the mean value of this outcome is not the difference between mean time spent in hospital and in ICU, since some patients die in ICU (and mortality depends on experiencing or not renal disease). Risk of renal disease in patients with low DO2 during CPB results more than double 


\begin{tabular}{lccl}
\multicolumn{1}{c}{ Parameters } & High DO2 & Low DO2 & Source \\
\hline Length of stay (days) [mean(SD)] & & & \\
Total in hospital & $12.4(12.1)$ & $17.6(14.1)$ & {$[1]$} \\
• in ICU & $2.5(4.4)$ & $4.2(8.7)$ & {$[1]$} \\
- in ward & $10.2(11.4)$ & $13.9(11.2)$ & Elaborated from hospital/ICU LOS according to \\
& & & mortality and AKI rate (see Appendix C) \\
Renal disease - AKI (\%) & 12.1 & 29.8 & Reported in [3], elaborated from [1] \\
- of which needing RT & 9.9 & 18.8 & RT rate reported in [2] rescaled to total AKI rate \\
\hline
\end{tabular}

Table III. Clinical input values depending on DO2 level

the risk in patients reaching the target $[1,3]$. The need for replacement therapy among patients experiencing AKI, elaborated from [2], results about $10 \%$ in patients reaching the DO2 target, while it is almost $20 \%$ in patients not reaching the target. Altogether, this implies the hypothesis that low DO2 is associated with more frequent and also more severe renal injury.

Costs were elaborated in a previous analysis on patients undergoing aortic valve replacement (AVR) [7]. All of the data are the national average costs for Medicare discharges associated with ICD-9-CM procedure code 35.22 for fiscal year (FY) 2013 and does not include Medicare Advantage health maintenance organizations (HMO) utilization. The national costs were calculated using the national average cost-to-charge ratios for $19 \mathrm{de}$ partments published by Medicare in the 2015. Statistical outliers were removed at the DRG level. Average length of stay overall and broken out by routine and ICU beds is included. The cost for a hospital stay was made up of the cost of the ICU in which the patient spends the days immediately after the surgical procedure plus the cost of the standard ward which hosts the patient after the critical postoperative phase has been completed. The cost of hospital haemodialysis treatment was included as a complication cost for patients that developed renal failure. It was defined as the additional charges/day for continuous replacement therapy (CRT) in the ICU to avoid double counting in ICU costs. An official blood bag tariff was taken as a proxy of the real cost for the collection, transportation and

\begin{tabular}{lc}
\hline \multicolumn{1}{c}{ Cost items } & Unit cost \\
\hline Renal replacement therapy $(\$ /$ day $)$ & 978.10 \\
ICU (\$/day) & $1,303.45$ \\
Ward (\$/day) & 779.98 \\
PRC unit transfused $(\$)$ & 295.94 \\
\hline
\end{tabular}

Table IV. Unit costs used in the model storage of patient blood. Unit cost summarized in Table IV, (they were not updated since calculated using tariffs).

The cost of GDP strategy was calculated considering the introduction of Sorin Heartlink (HL) Card/GDP Card and Sorin Connect (electronic data management system); for every cost the worst case (i.e. higher possible price) was considered: cost of the card was \$ 150.00 for US and cost of Connect per patient, calculated dividing the total hospital cost for GDP Monitors (assuming three systems per hospital) by the number of cases per HL Card/GDP Card and the Connect lifetime (10 years), results in $\$ 29.87$.

\section{Simulation technique}

\section{Base-case}

The simulation technique used for the calculation of base-case results is a so-called micro-simulation or patient-level simulation. According to this technique, each iteration of the simulation represents a unique patient, which runs through both arms of the model (TP and GDP strategies). The parameters of each iteration are drawn from their distributions, allowing the representation of the individual variability in the outcomes of the simulated population. Patient-level parameters and their distributions are presented in Appendix D.

\section{Probabilistic sensitivity analysis}

While the micro-simulation takes into account the variability in the population, the probabilistic sensitivity analysis (PSA) allows considering the uncertainty on key parameters and its effect on the estimated outcomes. This is obtained by a two level Monte Carlo simulation: the inner loop (1,000 iterations) is the patient-level simulation, which is averaged and repeated 1,000 times (outer loop) to perform PSA on key model parameters. In the absence of reliable data on their uncertainty, we set a standard deviation of $20 \%$ of their mean value, and attributed adequate distributions according to the type of 
data (i.e. gamma distributions for costs data and beta distributions for probabilities). For each cost item, the mean value (depending on the selected country) was multiplied by a gamma distributed factor with mean 1 and SD equal to $20 \%$. The parameters on which the PSA is conducted and their distributions are presented in Appendix C.

\section{Deterministic sensitivity analysis}

A deterministic (one-way) sensitivity analysis (DSA) was performed in order to test the sensitivity of result to variations of base-case estimates. A variation of $\pm 20 \%$ was considered for each parameter except nadir HCT during CPB that varies between $-10 \%$ (to avoid unrealistic lower HCT values) and $+35 \%$ (to take into account higher HCT values reported in recent surgical series) and probability to achieve the DO2 goal $( \pm 5 \%)$ to avoid probabilities higher than $100 \%$.

\section{Further analyses}

Since the nadir HCT and the DO2 level are the center point of the simulation we studied the cost difference (GDP vs. TP strategy) in the worst case for GDP efficacy $(+35 \%$ in the distribution of nadir HCT) for different values of the probability to achieve the $\mathrm{DO} 2$ target with GDP for patients with HCT < $26 \%$ (such probability was varied between $20 \%$ and $90 \%$ ).

\section{RESULTS}

Outcomes and costs resulting from 10,000 simulated patients are shown in Table $\mathrm{V}$ and Table VI as mean and 95\% confidence interval (calculated from PSA).

In the GDP strategy more than $90 \%$ of patients reach the DO2 target while in TP strategy less than $30 \%$. Renal disease incidence is lower with respect to traditional perfusion both in terms of AKI episodes (11\% AKI episodes saved) and need for RRT ( $2.5 \%$ dialysis procedures saved). Reduction in operative mortality (3.6 with TP vs $2.5 \%$ with GDP) causes the small increment in PRCs transfused (post CPB) with GDP strategy; this is due to the so called paradox effect: there are more patients alive in ICU which can be transfused. On average, GDP strategy saves more than 3 days in hospital, of which about 1 ICU day.

Total cost with TP results in about $\$ 15,300$ while GDP reduces the total cost by more than $\$ 3,000$. For both strategies the main driver is the hospital cost, of which almost one third is due to ICU stay. Furthermore, the cost of GDP strategy (Sorin GDP ${ }^{\mathrm{TM}}$ Monitor and Sorin Connect ${ }^{\mathrm{TM}}$ ) is completely offset by the saving in hospital stay (more than \$3,000).

Main outcomes reduction, hospital and total savings result statistically significant since the $95 \%$ confidence intervals do not cross the zero boundary. Choosing hospital stay reduc-

\begin{tabular}{lccc}
\hline Outcome [Mean $\mathbf{( 9 5 \%} \mathbf{~ C I )}$ & TP strategy & GDP strategy & Delta (GDP vs. TP) \\
\hline DO2 target achieved (\%) & $26.9(19.6-35.4)$ & $91.4(64.8-100.0)$ & $-64.4(-93.2--36.4)$ \\
Hospital stay (days) & $16.15(15.05-17.05)$ & $12.77(11.22-14.36)$ & $3.38(1.53-4.99)$ \\
- ICU stay (days) & $3.63(3.16-4.16)$ & $2.52(2.06-3.14)$ & $1.11(0.40-1.72)$ \\
AKI episode (\%) & $24.5(15.7-34.5)$ & $13.6(6.5-20.7)$ & $10.9(1.3-21.8)$ \\
- Need for RRT (\%) & $4.2(1.8-7.1)$ & $1.7(0.0-3.1)$ & $2.5(0.2-5.7)$ \\
Operative mortality (\%) & $3.6(1.8-5.9)$ & $2.5(1.1-4.1)$ & $1.1(-0.2-2.7)$ \\
PRC transfused (n.) & $2.48(2.0-2.92)$ & $2.49(2.0-2.93)$ & $0.01(0.00-0.01)$ \\
\hline
\end{tabular}

Table V. Comparison between traditional and goal directed perfusion: mean results from base case (10,000 simulated patients) while the $95 \% \mathrm{Cl}$ is calculated from PSA $(1,000 \times 1,000$ simulation $)$

\begin{tabular}{lccc}
\hline & & Costs (\$) [Mean (95\% CI)] & Savings (GDP vs TP) \\
\cline { 2 - 4 } & TP strategy & GDP strategy & $3,222(1,235-4,950)$ \\
\hline Hospital stay & $14,499(10,098-18,755)$ & $3,276(7,607-15,060)$ & $1,452(357-2,403)$ \\
- ICU stay & $4,737(2,773-6,772)$ & $37(-28-114)$ & $97(-39-294)$ \\
Renal complication & $134(1-340)$ & $737(443-1,025)$ & $-3(-7-1)$ \\
Transfusion & $734(441-1,021)$ & $180(113-249)$ & $-180(-249-113)$ \\
GDP monitor* & NA & $\mathbf{1 2 , 2 3 0}(\mathbf{8 , 5 2 8 - 1 6 , 0 5 5 )}$ & $\mathbf{3 , 1 3 7}(\mathbf{1 , 1 2 2 - 4 , 9 5 1 )}$ \\
Total cost & $\mathbf{1 5 , 3 6 7}(\mathbf{1 0 , 9 8 5 - 1 9 , 6 7 0 )}$ & &
\end{tabular}

Table VI. Resulting cost for traditional and goal directed perfusion: mean results from base case (10,000 simulated patients) while the $95 \% \mathrm{Cl}$ is calculated from PSA (1,000 $\times 1,000$ simulation)

*HL Card/GDP Card+Connect 


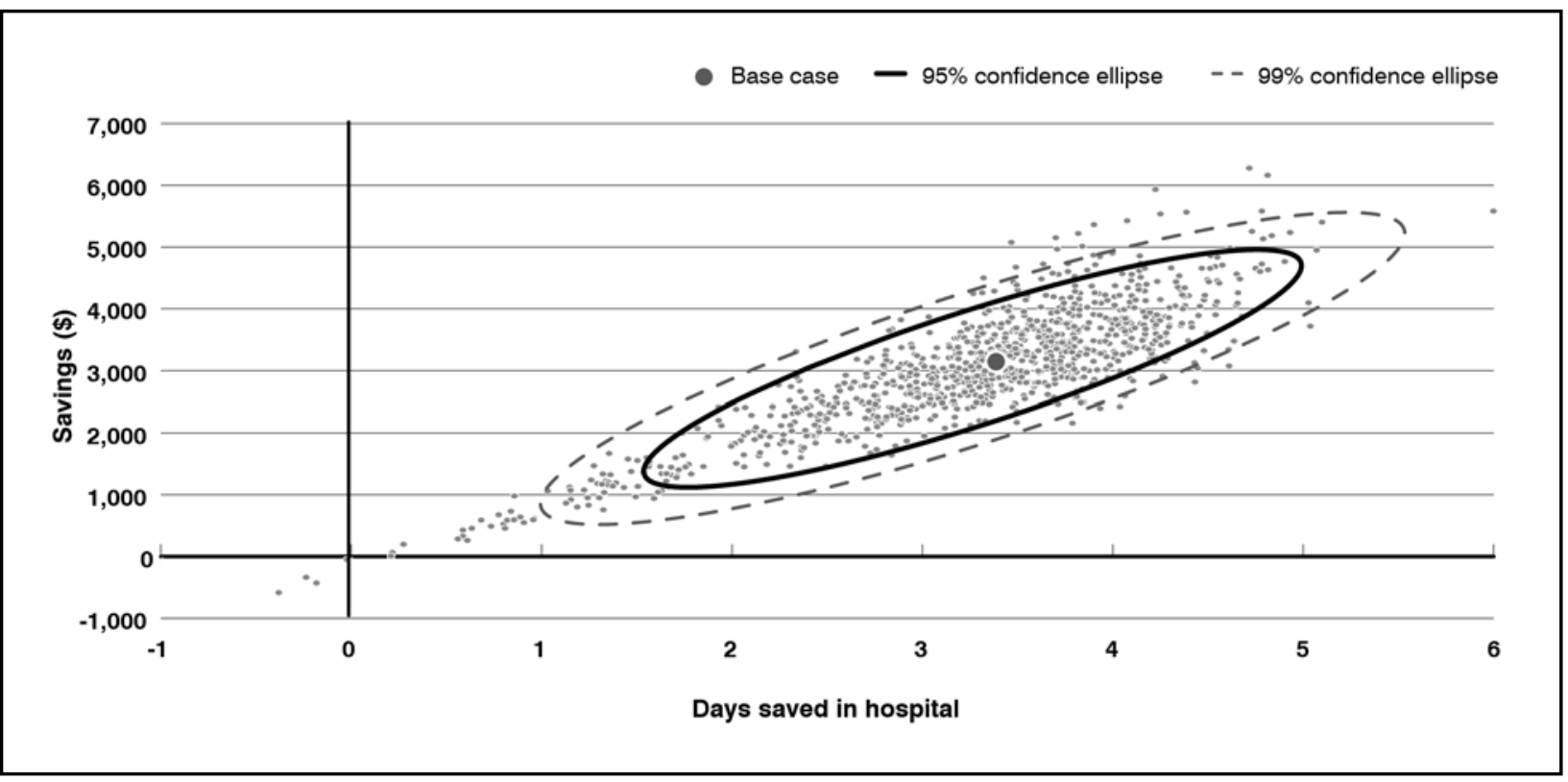

Figure 1. Cost-effectiveness plane (GDP vs. TP strategy): red point represent base case simulation result while light blue points are the 1,000 simulation in the PSA; continuous and dashed lines represent $95 \%$ and $99 \%$ confidence ellipses, respectively

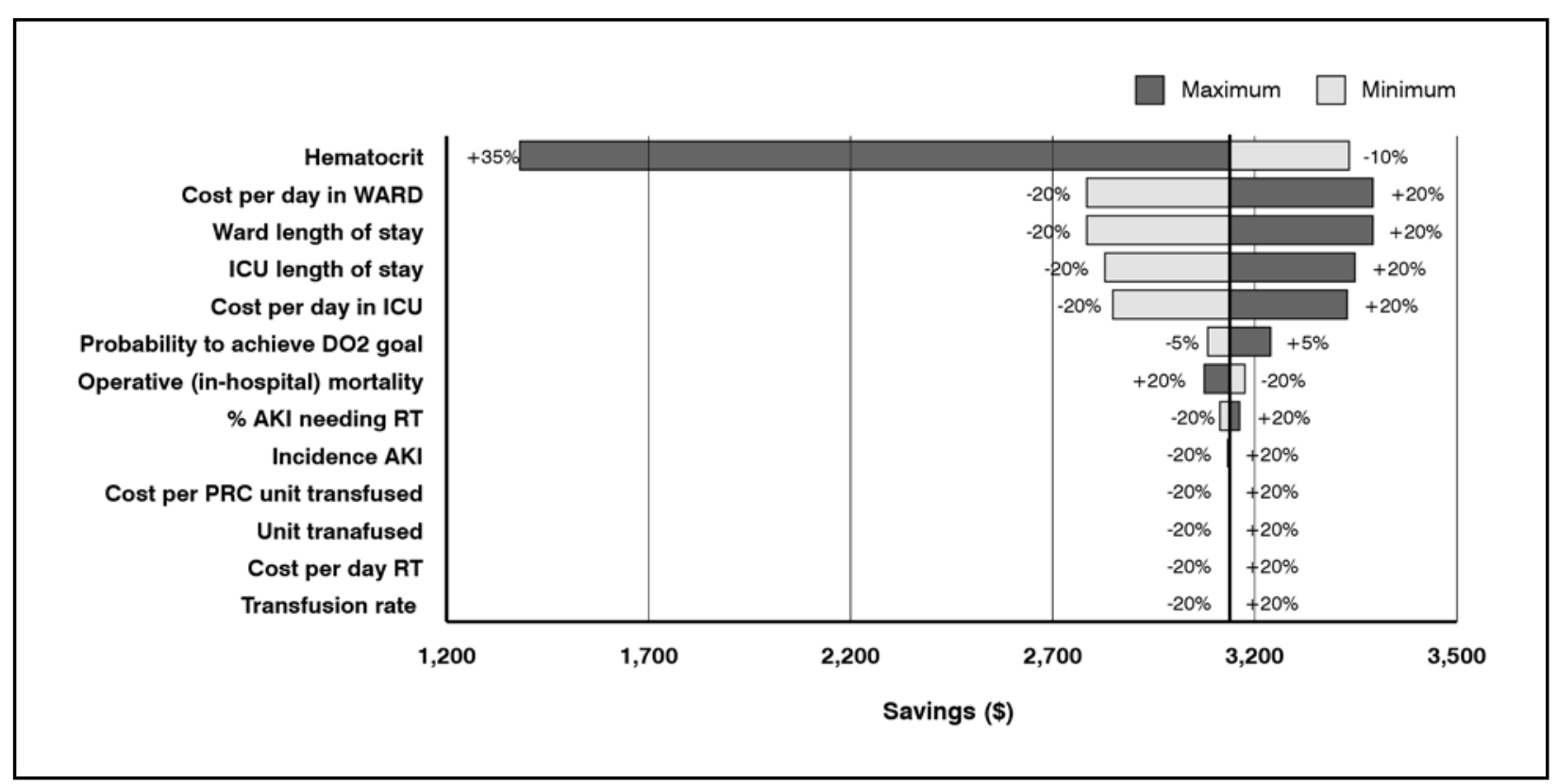

Figure 2. Deterministic sensitivity analysis - tornado diagram for saving in US (GDP vs. TP strategy)

tion as effectiveness measure, GDP strategy results cost-effectiveness in more than $95 \%$ of cases (Figure 1). In particular both $95 \%$ and $99 \%$ confidence ellipses lie in the first quadrant of the cost-effectiveness plane (less hospital stay and more cost savings), completely.

Total saving is mostly influenced by nadir haematocrit and, to a lesser extent, by hospital length of stay and hospital cost per day, both in ICU and ward (Figure 2). In particular DSA results show that GDP is more costsaving for patients with lower nadir HCT, for longer hospital stays and in structures with higher hospital costs.
Analysis on the probability to achieve the DO2 target shows that GDP strategy remains cost-saving also with worse hypothesis of GDP efficacy in a population with higher nadir HCT values (Figure 3). In the worst case, with a $20 \%$ probability of success of GDP strategy, a saving of almost $\$ 400$ per case is still possible.

\section{DISCUSSION}

Innovation in the delivery of modern healthcare is challenging and profitable; a win-win situation where efficient outcomes are encountered, is valuable and infrequent. 


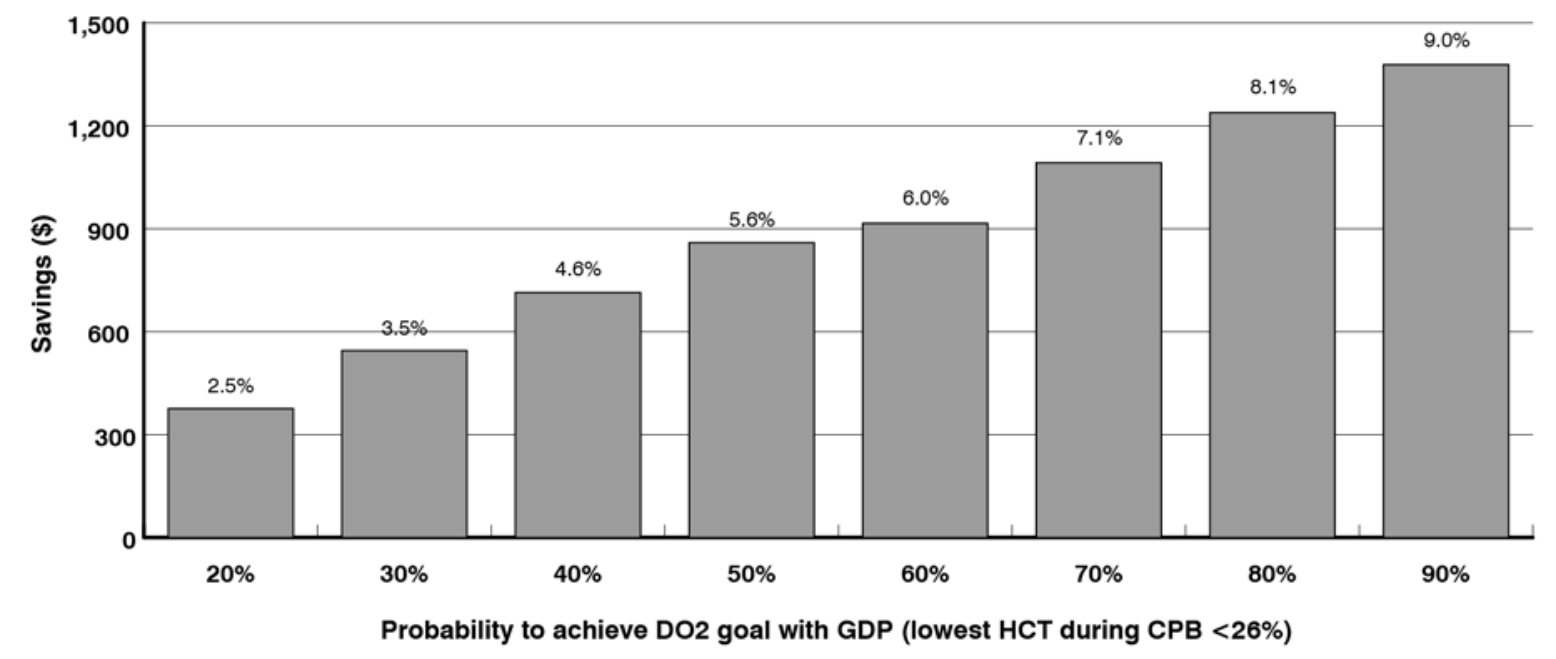

Figure 3. Savings with respect to different hypothesis of GDP efficacy to achieve DO2 target during CPB in low HCT patients in the worst case for GDP efficacy (+35\% for nadir HCT distribution). Light red bar represents saving with GDP base case efficacy

Just by delivering the right information at the right time during surgery, directing perfusion decisions, undesirable and costly clinical outcomes could be avoided. Assuming that with GDP, compared to TP, the probability to achieve DO2 goal would dramatically increase, on average $\$ 3,137$ per patient $(95 \%$ CI: $1,122-4,951)$ could be saved. On average 3.38 ward days (95\% CI: $1.53-4.99)$ and $1.11 \mathrm{ICU}$ days (95\% CI: $0.40-1.72$ ) would be avoided per patient. Hence, the intuitive cost driver, intervention cost, was offset mainly by the avoided costs of hospital stay. Reducing the incidence of AKI episodes, and then the need for RRT, also impacted the encountered savings but to a much lesser extent.

Presented sensitivity analysis confirms the cost-effectiveness of GDP. In the DSA, when input parameters were varied to a minimum and a maximum value, the total savings per patient remained in favour of GDP. This savings was more sensitive by the HCT values as the expected gain is expected to be more with those lower HCT values. Then, ward and ICU stays/costs also impacted the amount of savings. All other input clinical parameters like probability of achieving DO2 targets or hospital mortality do not seem to impact the economic outcomes. Similiarly, assumed cost drivers related to AKI or PRC unit perfusion have ignorable impact. Furthermore, in order to invistigate the relationship between the probability of achieving DO2 goal with GDP when HCT distribution is assumed to be $35 \%$ above the base case value: the expected benefit is lower $(\$ 1,378)$, we varied this probability from $90 \%$ to as low as $20 \%$ and the cost savings was still realizable.
In summary, savings on the microlevel, patient level or operating theater level, or macrolevel (regional or national) could be considerable.

Estimated results seem comparable with previous economic analyses. A recent study [8], analyzing 953 patients with diabetes mellitus and multivessel coronary artery disease undergoing $\mathrm{CABG}$ with standard techniques, estimates a total cost of about $\$ 19,500$ (excluding precedural costs and physician fees not considered in this analysis); value calculated in [8] is higher since it includes also ancillary services. In a less recent paper [9], the direct costs for 4698 patients undergoing CABG at 5 US hospital amount to $\$ 12,800$; a lower results may be due to a shorter hospital LOS (8.7 days). Other two studies reported a mean cost (excluding physician fees) of $\$ 15,713$ [10] and \$ 20,574 [11] (2000 US dollars).

\section{CONCLUSION}

Few interventions have positive impact on clinical outcomes and costs. This economic forecast suggests that even when the probability of achieving DO2 goal with GDP is challenged below plausible values, GDP remains a cost-effective strategy. Thus, early uptake of such innovative intervention is endorsed until such benefits are empirically quantified and cost savings are realized. Furhtermore, saving is mostly influenced by hospital LOS, cost per day both in ICU and in ward, and nadir HCT during CPB and additional costs due to perform GDP strategy have no impact on the total cost since completely offset by the savings in hospital cost. 


\section{REFERENCES}

1. de Somer F, Mulholland JW, Bryan MR, et al. O2 delivery and CO2 production during cardiopulmonary bypass as determinants of acute kidney injury: time for a goal-directed perfusion management? Crit Care 2011; 15: R192; http://dx.doi.org/10.1186/cc10349

2. Ranucci M, Romitti F, Isgrò G, et al. Oxygen delivery during cardiopolmunary bypass and acute renal failure after coronary operation. Ann Torac Surg 2005; 80: 2213-20; http://dx.doi.org710.1016/j.athoracsur.2015.02.034

3. The Goal dIrected perFusion Trial in Cardiac Surgery (GIFT). ClinicalTrials.gov Identifier: NCT02250131

4. Habib R, Zacharias A, Schwann T, et al. Adverse effects on low hematocrit during cardiopulmonary bypass in the adult: Should current practice be change? J Thorac Cardiovasc Surg 2003; 125: 1438-50; http://dx.doi.org/10.1016/ S0022-5223(02)73291-1

5. Karkouti K, Wikeysundera DN, Yau T et al. Acute Kidney Injury After Cardiac Surgery: Focus on Modifiable Risk Factors. Circulation 2009; 119: 495-502; http://dx.doi.org/10.1161/circulationaha.108.786913

6. Pradelli L, Zaniolo O. Perceval Sutureless valves in isolated and concomitant AVR procedures: an economic model shows overall decrease of costs for isolated or combined operations. Farmeconomia. Health economics and therapeutic pathways 2012; 13: 159-174; http://dx.doi.org/10.7175/fe.v13i4.282

7. Average Medicare Cost for AVR Patient. MedPar Report, 2013

8. Magnuson EA, Farkouh ME, Fuster V, et al. Cost-effectiveness of percutaneous coronary intervention with drug eluting stents versus bypass surgery for patients with diabetes mellitus and multivessel coronary artery disease - Results from the FREEDOM trial. Circulation 2013; 127: 820-31; http://dx.doi.org/10.1161/CIRCULATIONAHA.112.147488

9. Eisenberg MH, Filion KB, Azoulay A, et al. Outcomes and cost of coronary artery bypass graft surgery in the United States and Canada. Arch Intern Med 2005; 165: 1506-13; http://dx.doi.org/10.1001/archinte.165.13.1506

10. Cowper PA, DeLong ER, Peterson ED, et al. Variability in cost of coronary bypass surgery in New York State: potential for cost savings. Am Heart J 2002; 143: 130-9; http://dx.doi.org/10.1067/mhj.2002.119617

11. Reynolds MR, Neil N, Ho KK, et al. Clinical and economic outcomes of multivessel coronary stenting compared with bypass surgery: a single-center US experience. Am Heart J 2003; 145: 334-42

12. Mehta RL, Kellum JA, Shah SV, et al. and the Acute Kidney Injury Network. Acute Kidney Injury Network: a report of an initiative to improve outcomes in acute kidney injury. Crit Care 2007; 11: R31; http://dx.doi.org/10.1186/cc5713

13. Cockcroft D, Gault MD. Prediction of creatinine clearance from serum creatinine. Nephron 1976; 16: 31-41; http:// dx.doi.org/10.1159/000180580

\section{APPENDIX A}

\section{The DO2 formula}

DO2 is the amount of oxygen delivered to the whole body from the lungs/oxygenators. It is defined by the formula

$$
\mathrm{DO} 2=10 \times \mathrm{Qb} \times\left(1.36 \times \frac{\mathrm{HCT}}{2.94} \times \mathrm{S}_{\mathrm{a}} \mathrm{O}_{2}+0.003 \times \mathrm{p}_{\mathrm{a}} \mathrm{O}_{2}\right)
$$

where $\mathrm{Qb}$ is the total blood flow or cardiac output, HCT is the haematocrit level, is the arterial saturation, is the arterial tension and 0.003 is the solubility coefficient of oxygen in human plasma.

\section{APPENDIX B}

\section{Relation between serum creatinine and eGFR}

In the GIFT protocol, AKI is defined according to AKIN criteria [12] as:

$$
\text { EQ } 1 \quad \mathrm{Sc}_{\mathrm{PO}}>1.5 \mathrm{Sc}_{\mathrm{B}}
$$


where $\mathrm{Sc}_{\mathrm{PO}}$ is the post-operative serum creatinine and $\mathrm{Sc}_{\mathrm{B}}$ is the value at baseline. In [5] AKI is defined as a decrease greater than $50 \%$ in eGFR within one week of surgery, that is:

$$
\text { EQ } 2 \frac{\text { eGFR(B) }-\mathrm{eGFR}(\mathrm{PO})}{\mathrm{eGFR}(\mathrm{B})}>50 \%
$$

where eGFR is calculated at baseline (B) and post CPB (PO). We find the relation between definitions (EQ 1) and (EQ 2); since eGFR in [5] is estimated using the Cockcroft-Gault formula [13] we have:

$$
\text { EQ } 3 \text { eGFR }=\frac{(140-\text { Age }) \times \mathrm{W}}{72 \times \mathrm{Sc}} \times(0.85 \text { if female })
$$

where $\mathrm{Sc}$ is the serum creatinine valued expressed in $\mathrm{mg} / \mathrm{dl}$, the age is measured in years and $\mathrm{W}$ is the real weight if $\mathrm{BMI}$ is normal (between 18.5 and 25) otherwise is calculated as the ideal BMI (18.5 if BMI $<18.5$ and 25 if $\mathrm{BMI}>$ 25) multiplied by the square of height.

For each patient, we can consider age and weight constant since the observational time is about a week, than (EQ 3) is simply:

$$
\text { EQ } 4 \quad \text { eGFR }=\frac{\mathrm{A}}{\mathrm{Sc}}
$$

where the constant A contains all information on age, weight and sex.

Using definition (EQ 4), we can re-write (EQ 2) for a general reduction $\beta$ :

$$
\begin{gathered}
\mathrm{Sc}_{\mathrm{PO}}>\frac{\mathrm{Sc}_{\mathrm{B}}}{1-\beta} \\
\frac{\mathrm{Sc}_{\mathrm{PO}}-\mathrm{Sc}_{\mathrm{B}}}{\mathrm{Sc}_{\mathrm{PO}}}>\beta \\
\mathrm{Sc}_{\mathrm{PO}}-\mathrm{Sc}_{\mathrm{B}}>\beta \mathrm{Sc}_{\mathrm{PO}} \\
\frac{\mathrm{A} / \mathrm{Sc}_{\mathrm{B}}-\mathrm{A} / \mathrm{Sc} \mathrm{PO}_{\mathrm{P}}}{\mathrm{A} / \mathrm{Sc}_{\mathrm{B}}}>\beta
\end{gathered}
$$

If we want, we have to fix $\beta=33 \%$.

Using this threshold we can calculate mortality rate for patient experiencing and not experiencing AKI from analysis reported in [5].

\begin{tabular}{ccccc}
\hline Decrease in eGFR (\%) & Deaths (n.) & Patients (n.) & AKI episode & Mortality rate (\%) \\
\hline$<25 \%$ & 25 & 2631 & NO & $\mathbf{1 . 2}$ \\
$25-50 \%$ & 25 & 601 & & \\
$25-33 \%$ & 8 & 200 & YES \\
$33-50 \%$ & 17 & 401 & \\
$50-75 \%$ & 12 & 109 & & $\mathbf{1 1 . 9}$ \\
$>75 \%$ & 46 & 119 & & \\
\hline
\end{tabular}

Table IA. Elaboration of post-operative mortality based on [5]

\section{APPENDIX C}

\section{Calculation of the length of stay distribution in ward (post ICU)}

For each simulated patient, the total time spent in hospital is defined as the sum of LOS in ICU and LOS in ward (if patient was discharged alive from ICU). Furthermore, according to our model, mortality in ICU depends on experiencing an AKI episode. Figure 1A shows the decisional tree for calculation of hospital LOS, the structure is the same both for patient achieving and not achieving the DO2 target. 


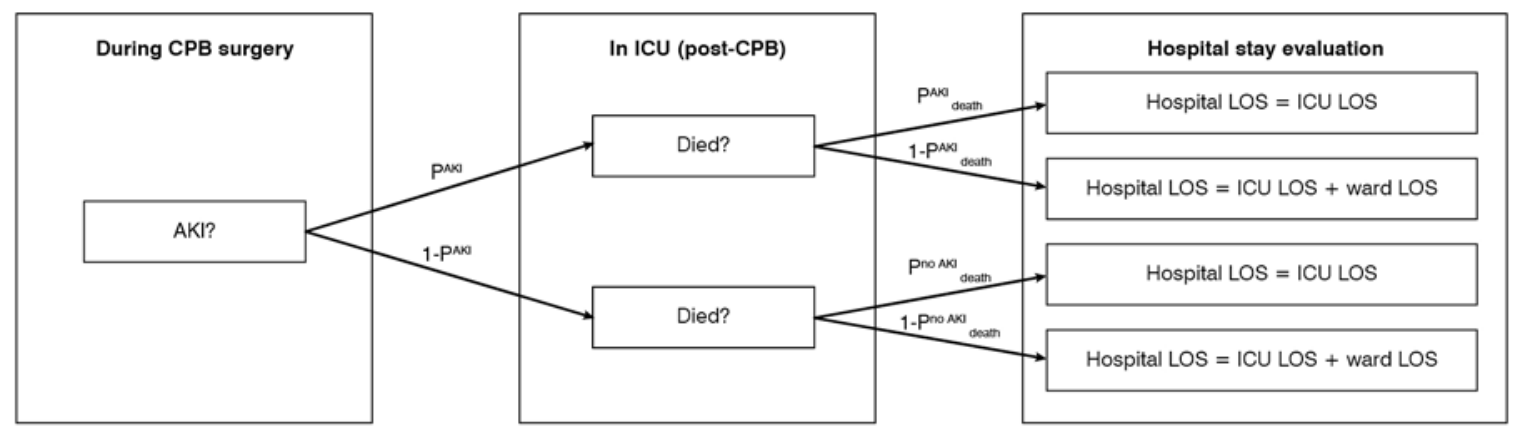

Figure 1A. Decisional tree for calculation of hospital length of stay

The distribution of LOS in ward was calculated using bootstrap method:

- 10,000 patients were simulated (both with DO2 target achieved and not achieved) sampling AKI rate, mortality and LOS in ICU from distributions fitted on data reported in Table I and Table II.

- Time spent in ward was sampled from a gamma distribution with parameters a and $\beta$ unknown.

- $a$ and $\beta$ was calculated imposing that the resulting hospital LOS distribution was a gamma distribution fitted on mean and SD reported in Table II.

\section{APPENDIX D}

\section{Parameter distributions}

\begin{tabular}{lccccc}
\hline & Mean & SD & $\alpha$ & $\lambda^{*}$ & Distribution \\
\hline Haematocrit & 21.4 & 4.2 & 20.19 & 74.16 & Beta \\
ICU length of stay & & & & & Gamma \\
High DO2 & 2.5 & 4.4 & 0.32 & 1.74 & Gamma \\
Low DO2 & 4.2 & 8.7 & 0.23 & & Gamma \\
Ward length of stay & & & & 12.63 & Gamma \\
High DO2 & 10.39 & 11.45 & 0.82 & 8.30 & Gamma \\
Low DO2 & 14.60 & & 1.76 & 2.35 & Gamma \\
Unit transfused & 1.7 & 2.0 & 0.72 & 1.80 & \\
During CPB & 1.1 & 1.4 & 0.60 & & \\
Post CPB & & & & & \\
\hline
\end{tabular}

Table IIA. Parameters and distribution sampled in the base case simulation (patients-level parameters)

*For beta distribution, the second parameters is usually named $\beta$

\begin{tabular}{|c|c|c|c|c|c|c|c|c|c|}
\hline \multirow{2}{*}{$\begin{array}{l}\text { Prob. to achieve } \\
\text { DO2 goa }\end{array}$} & Mean & SD & $\alpha$ & $\beta$ & Mean & SD & $\alpha$ & $\beta$ & \multirow{2}{*}{ Distribution } \\
\hline & \multicolumn{4}{|c|}{ TP strategy } & \multicolumn{4}{|c|}{ GDP strategy } & \\
\hline $\mathrm{HCT}>26 \%$ & 85 & 17 & 2.90 & 0.51 & & & & & beta \\
\hline \multirow[t]{2}{*}{ HCT $<26 \%$} & 18 & 3.6 & 20.32 & 92.57 & 90 & 18 & 1.60 & 0.18 & beta \\
\hline & & \multicolumn{3}{|c|}{ High DO2 } & & \multicolumn{3}{|c|}{ Low DO2 } & \\
\hline Incidence AKI (\%) & 12.1 & 2.42 & 21.85 & 158.76 & 29.8 & 5.96 & 17.25 & 40.64 & beta \\
\hline \% AKI needing RT & 9.9 & 1.98 & 22.43 & 204.10 & 18.8 & 3.76 & 20.11 & 86.87 & beta \\
\hline Transfusion rate (\%) & & \multicolumn{3}{|c|}{ During CPB } & & \multicolumn{3}{|c|}{ Post CPB } & \\
\hline $\mathrm{HCT}<24 \%$ & 50 & 10 & 12.00 & 12.00 & 50 & 10 & 12.00 & 12.00 & beta \\
\hline \multirow[t]{2}{*}{$\mathrm{HCT}<30 \%$} & & & & & 25 & 5 & 18.50 & 55.50 & beta \\
\hline & & \multicolumn{3}{|c|}{ With AKI } & & \multicolumn{3}{|c|}{ w/o AKI } & \\
\hline Operative mortality (\%) & 25.4 & 5.08 & 18.39 & 53.89 & 1.5 & 0.31 & 24.60 & 1,565 & beta \\
\hline
\end{tabular}

Table IIIA. Parameters and distribution sampled in the PSA (outer loop parameters) 